\title{
Modeling exchange rate dependence dynamics at different time horizons
}

\author{
Alexandra Dias ${ }^{\mathrm{a}, *}$, Paul Embrechts ${ }^{\mathrm{b}, 1}$ \\ ${ }^{a}$ Warwick Business School, Finance Group, University of Warwick, CV4 7AL Coventry, \\ $U K$. \\ ${ }^{b}$ Department of Mathematics, ETH Zurich, 8092 Zurich, Switzerland.
}

\begin{abstract}
Despite an extensive body of research, the best way to model the dependence of exchange rates remains an open question. In this paper we present a new approach which employs a flexible time-varying copula model. It allows the conditional correlation between exchange rates to be both time-varying and modeled independently from the marginal distributions. We introduce a dynamic specification for the correlation using the Fisher transformation. Applied to Euro/US dollar and Japanese Yen/US dollar, our results reveal a significantly time-varying correlation, dependent on the past return realizations. We find that a time-varying copula with the proposed correlation specification gives better results than alternative dynamic benchmark models. The dynamic copula model outperforms at six different time horizons, ranging from hourly to daily, confirming the model specification.
\end{abstract}

JEL Classification: C32, C50, F31

Keywords: Foreign exchange rates, Multivariate time series, Copula-GARCH, Conditional dependence, Dynamic copula

\footnotetext{
*Corresponding author. Tel.: +44(0) 247657 4297; fax: +44(0) 2476523779.

Email address: alexandra.dias@wbs.ac.uk (Alexandra Dias)

${ }^{1}$ Paul Embrechts acknowledges financial support from the Swiss Finance Institute.
} 


\section{Introduction}

In economics and finance multivariate problems are often of interest in areas like risk management, asset pricing, portfolio allocation and forecasting. There, the specification of financial variables' multivariate distributions is crucial for the computation of quantities related to their inter-dependence. The prime example is the linear correlation, the perfect measure of dependence for multivariate normal variables and to some extent for elliptical variables. However there is strong evidence that the univariate distributions of many financial variables are non-normal and significantly fat-tailed. This empirical fact often rules out the use of the multivariate normal distribution. In principle, there is no reason for different marginal variables to have the same degree of fat-tailedness or even to have univariate distributions of the same type. Moreover, most financial data exhibit skewness. This also questions the use of elliptical distributions in other contexts.

Financial time-series are often modeled with GARCH type models. In the multivariate GARCH literature there exist several models, like CCCGARCH, DVEC, matrix-diagonal GARCH, BEKK and principal components GARCH. ${ }^{2}$

In all these models the conditional multivariate distribution is Gaussian

\footnotetext{
${ }^{2}$ For a survey on multivariate GARCH models see Bauwens et al. (2006).
} 
or Student-t. An alternative approach to overcome that distributional constraint is to use copula-GARCH models; see Jondeau and Rockinger (2006), Fortin and Kuzmics (2002), Patton (2006a) and Patton (2006b). ${ }^{3}$ Copula based models allow complete freedom to combine different conditional marginal distributions in a dependence structure.

By construction, the conditional variance of the univariate distributions in a GARCH model is time-varying. Additionally, in the copula-GARCH model the parameters of the dependence structure can also be time-varying. This is potentially useful as there is evidence that asset's dependence is timevarying, a fact that has raised considerable interest in the dynamic behavior of correlation between different risks as a function of time; see for instance Boyer et al. (1999), Engle (2009), Longin and Solnik (2001), Loretan and English (2000) and Loretan and Phillips (1994). Because of the fundamental importance of the notion of linear correlation in finance and insurance, such dynamics may have a non-trivial impact on the pricing and hedging of underlying instruments, or on the risk measurement of such positions. As a consequence, a systematic modeling of the dynamic behavior of the dependence structure underlying multivariate variables is of considerable importance. This can be achieved with time-varying copula-GARCH models.

\footnotetext{
${ }^{3}$ Another possible alternative, which we opt not to pursue here, is to model regime changes. In the context of time series analysis, see for instance Hamilton (1990).
} 
In the present article we present a time-varying copula-GARCH model. We propose a specification for the dynamics of the dependence parameter using the Fisher transformation in order to model the dynamic dependence between Euro and Japanese Yen versus U.S. Dollar exchange rates. With copula-GARCH models we allow for possibly different fat-tailed univariate distributions. We look for the most appropriate copula family for both timeinvariant and time-varying copula models.

We compare the proposed model with two competing models: the dynamics proposed by Tse and Tsui (2002) and the BEKK model introduced by Engle and Kroner (1995). We evaluate the estimated dependence paths by comparing them with two benchmark non-parametric measures of dependence: the realized correlation and Kendall's tau. Finally we investigate six time horizons ranging from hourly to daily. Our goal is to examine the consistency of our findings across different time horizons. Theoretical properties of time aggregation of univariate GARCH models can for instance be found in Drost and Werker (1996) and Meddahi and Renault (2004).

The paper is organized as follows. In Section 2 we introduce the timevarying specification for copula-GARCH models and its estimation methodology. In Section 3 we describe the data and the computation of the bivariate returns on the Euro and Japanese Yen spot rates, both quoted against the U.S. Dollar. These are deseasonalized bivariate returns at six time horizons 
from one hour to daily. The first step of the copula-GARCH modeling is performed in Section 4. This consists of univariate GARCH filtering and consequent specification tests. In Section 5 we estimate the time-varying dependence paths using several dynamic copula models, including the time-varying copula dynamics introduced by Tse and Tsui (2002) and the time-varying correlation implied by a BEKK model. The evaluation of the estimated timevarying dependence paths against realized correlation and Kendall's tau is given in Section 6. Our results are summarized in Section 7 which concludes the paper.

\section{The model specification and estimation}

We proceed to the specification of the dynamic copula model starting by introducing the general copula-GARCH type model.

\subsection{The copula-GARCH type model}

We denote the observed bivariate time series of the exchange rate returns by $\left(\mathbf{x}_{t}\right)=\left(x_{1 t}, x_{2 t}\right)^{\prime}, t=1, \ldots, T$ and model these data with a copula-AR$\mathrm{GARCH}^{4}$ specification as follows. Each of the returns' univariate processes

\footnotetext{
${ }^{4}$ We considered also copula-ARMA-GARCH models but the moving average dynamics is not significant for the FX data analyzed here.
} 
satisfies

$$
\begin{aligned}
X_{i t} & =\mu_{i t}+\epsilon_{i t}, \quad \mu_{i t}=\mu_{i}+\sum_{l=1}^{r} \phi_{i l}\left(X_{i t-l}-\mu_{i}\right), \\
\epsilon_{i t} & =\sigma_{i t} Z_{i t}, \\
\sigma_{i t}^{2} & =\alpha_{i 0}+\sum_{k=1}^{p} \alpha_{i k} \epsilon_{i t-k}^{2}+\sum_{j=1}^{q} \beta_{i j} \sigma_{i t-j}^{2}, \quad i=1,2,
\end{aligned}
$$

where $\phi_{i l}$ are autoregressive coefficients, the parameters $\alpha_{i 0}, \alpha_{i k}$ and $\beta_{i j}$ are non-negative, and $\sum \alpha_{i k}+\sum \beta_{i j}<1$. The innovations $Z_{i t}$ are independent of $\left(X_{i s}\right)_{s \leq t}$, have mean zero, unit variance, and bivariate distribution function $F$ with continuous univariate marginal distribution functions $F_{1}$ and $F_{2}$. The bivariate innovations are characterized by a copula-based model

$$
F\left(z_{1}, z_{2} ; \boldsymbol{\theta}_{t}\right)=C\left(F_{1}\left(z_{1}\right), F_{2}\left(z_{2}\right) ; \boldsymbol{\theta}_{t}\right),
$$

where $C$ is a copula function which, as long as the marginal distribution functions are continuous, we know to exist uniquely by Sklar's Theorem (Sklar (1959)). We suppose the copula $C$ is parameterized by the vector $\boldsymbol{\theta}_{t} \in \mathbb{R}^{q}$ with $q \in \mathbb{N}$.

\section{A dynamic copula model}

The search for the most appropriate dependence parameter dynamics depends strongly on the interpretation that a specific (copula) dependence parameter may have. We shall see in Section 5 that the Gaussian and the t-copula yield the best models for the dependence structure of the Euro and 
Japanese Yen returns. For that reason we focus here on the dynamics for these copulae parameters.

Let $\rho_{t}$ be the correlation parameter of a Gaussian or $t$-copula at time $t$. Given the coefficients $\alpha, \beta$ and $\gamma$, set

$$
\rho_{t}=h^{-1}\left(\alpha+\beta \operatorname{sign}\left(Z_{1 t-1} Z_{2 t-1}\right)\left|Z_{1 t-1} Z_{2 t-1}\right|^{1 / 2}+\gamma h\left(\rho_{t-1}\right)\right),
$$

where $h(\cdot)$ is Fisher's transformation

$$
h(\rho)=\log \left(\frac{1+\rho}{1-\rho}\right), \quad-1<\rho<1,
$$

$Z_{i t}$ are the innovations in $(1)$ and $\gamma<1$. We use $\rho_{t}$ as our dynamic correlation parameter. Throughout the paper we call specification (3) the Fisher dynamics. This specification ensures that $\rho_{t}$ is between -1 and 1 . As a consequence of the regression-type dynamics of the innovations $z_{i t}$ in (3), when both innovations have the same sign we have a positive contribution to the correlation. When innovations have opposite signs, a negative contribution results. This agrees with evidence found by other authors that dependence is stronger when returns have the same sign than when returns have opposite signs; see for instance Jondeau and Rockinger (2006). We do not split the dynamics in the case of both positive and both negative returns because, according to the tests in Section 5, there is no evidence of asymmetry in the dependence structure for these data. 
Specifications evolving from the vech-diagonal model introduced by Bollerslev et al. (1988) typically assume a time-varying covariance dynamics similar to the third line equation in (1), where $\rho_{t}$ replaces $\sigma_{i t}^{2}$ and $\epsilon_{i t} \epsilon_{j t}$ replaces $\epsilon_{i t}^{2}$. In this setting we consider, for comparison with our model, the time-varying correlation dynamics proposed by Tse and Tsui (2002). Tse and Tsui (2002) define a dependence parameter $\rho_{t}$ satisfying the equation:

$$
\rho_{t}=(1-\beta-\gamma) \rho+\beta \xi_{t-1}+\gamma \rho_{t-1}
$$

where $\beta$ and $\gamma$ are non-negative, $\beta+\gamma \leq 1$, and

$$
\xi_{t}=\frac{\sum_{h=0}^{m-1} z_{1 t-h} z_{2 t-h}}{\sqrt{\sum_{h=0}^{m-1} z_{1 t-h}^{2} \sum_{h=0}^{m-1} z_{2 t-h}^{2}}}
$$

with $m \geq 2$. In this paper we call specification (4) the Tse-Tsui dynamics. In the empirical study we use the Gaussian and the $t$-copula models each with Fisher dynamics and with Tse-Tsui dynamics.

Patton (2006b) introduces a model for the symmetrized Joe-Clayton copula parameters (upper and lower tail parameter), defined dynamically as

$$
\tau_{t}=\Lambda\left(\omega+\beta \tau_{t-1}+\alpha \frac{1}{10} \sum_{j=1}^{10}\left|F_{1}\left(z_{1 t-j}\right)-F_{2}\left(z_{2 t-j}\right)\right|\right)
$$

where the logistic transformation $\Lambda(x)=\left(1+e^{-x}\right)^{-1}$ keeps $\tau_{t}$ in its interval domain $(0,1)$. In the same paper, the Gaussian copula correlation parameter is modeled with dynamics similar to (5) where $\rho_{t}$ replaces $\tau_{t}$, $\Phi^{-1}\left(F_{1}\left(z_{1 t-j}\right)\right) . \Phi^{-1}\left(F_{2}\left(z_{2 t-j}\right)\right)$ replaces $\left|F_{1}\left(z_{1 t-j}\right)-F_{2}\left(z_{2 t-j}\right)\right|$ and $\tilde{\Lambda}(x)=$ 
$\left(1-e^{-x}\right)\left(1+e^{-x}\right)^{-1}$ replaces $\Lambda$, where $\Phi$ represents the standard normal distribution function.

The dynamics we propose for the correlation parameter differs from Tse and Tsui (2002) because we use the Fisher transformation to ensure the correlation estimates to be in $[-1,1]$ and because we only use one lag of the past observations. The Patton (2006b) dynamics are based on ten past observations. Additionally, we regress the Fisher transformed correlation on the lagged Fisher transformed correlation while in Patton (2006b) the transformed correlation is regressed on a non-transformed correlation.

The $t$-copula parameters are the degrees of freedom, $\nu$, and the correlation. We assume that the degrees of freedom are constant over time, as for the univariate margins, and allow the correlation to be time-varying. Recall that the innovations of the univariate or multivariate GARCH models, with Student- $t$ distributed innovations, have constant degrees of freedom even for those multivariate GARCH models which allow for time-varying correlation. A generalization of the procedure for time-varying $\nu_{t}$ is definitely possible for the copula as well as for the margins.

\subsection{Estimation}

For a random sample $\left(\mathbf{Z}_{1}, \mathbf{Z}_{2}, \ldots, \mathbf{Z}_{T}\right)$, the distribution of $\mathbf{Z}_{t}$ can be written as

$$
F\left(\mathbf{z}_{t} ; \boldsymbol{\alpha}_{1}, \boldsymbol{\alpha}_{2}, \boldsymbol{\theta}_{t}\right)=C\left(F_{1}\left(z_{1 t} ; \boldsymbol{\alpha}_{1}\right), F_{2}\left(z_{2 t} ; \boldsymbol{\alpha}_{2}\right) ; \boldsymbol{\theta}_{t}\right)
$$


where we assume that each $F_{i}$ is absolutely continuous with density $f_{i}$; the vectors $\boldsymbol{\alpha}_{i}$ parameterize the marginal distribution functions and the timevarying parameter $\boldsymbol{\theta}_{t}$ parameterizes the copula family.

Assuming that $C$ has density $c$, given by

$$
c\left(u_{1}, u_{2} ; \boldsymbol{\theta}\right)=\frac{\partial^{2} C\left(u_{1}, u_{2} ; \boldsymbol{\theta}\right)}{\partial u_{1} \partial u_{2}}, \quad\left(u_{1}, u_{2}\right) \in[0,1]^{2},
$$

the density of $\mathbf{Z}_{t}$ is given by:

$$
f\left(\mathbf{z}_{t} ; \boldsymbol{\alpha}_{1}, \boldsymbol{\alpha}_{2}, \boldsymbol{\theta}_{t}\right)=c\left(F_{1}\left(z_{1 t} ; \boldsymbol{\alpha}_{1}\right), F_{2}\left(z_{2 t} ; \boldsymbol{\alpha}_{2}\right) ; \boldsymbol{\theta}_{t}\right) f_{1}\left(z_{1 t} ; \boldsymbol{\alpha}_{1}\right) f_{2}\left(z_{2 t} ; \boldsymbol{\alpha}_{2}\right) .
$$

The log-likelihood function of the model is therefore given by

$$
l\left(\boldsymbol{\alpha}_{1}, \boldsymbol{\alpha}_{2}, \boldsymbol{\theta}_{t}\right)=\sum_{t=p+1}^{T}\left(\log c\left(F_{1}\left(z_{1 t} ; \boldsymbol{\alpha}_{1}\right), F_{2}\left(z_{2 t} ; \boldsymbol{\alpha}_{2}\right) ; \boldsymbol{\theta}_{t}\right)+\sum_{i=1}^{2} \log f_{i}\left(z_{i t} ; \boldsymbol{\alpha}_{i}\right)\right),(6)
$$

with $p$ as in (1).

Numerical maximization of (6) yields the maximum likelihood estimates of the model. However, the optimization of the likelihood function with possibly many parameters is numerically difficult and time consuming. It is more tractable to estimate first the model parameters of the margins and then the dependence model parameters using the estimates from the first step. This two-step estimation procedure, also known as inference for margins (see for instance Joe (1997)) has been used in semi-parametric modeling, as in Andreou and Ghysels (2003), or in a full-parametric modeling context as in Engle and Sheppard (2001), Patton (2006b) and Jondeau and Rockinger 
(2006). The marginal log-likelihood functions

$$
l\left(\boldsymbol{\alpha}_{i}\right)=\sum_{t=p+1}^{T} \log f_{i}\left(z_{i, t} ; \boldsymbol{\alpha}_{i}\right), \quad i=1,2,
$$

are independently maximized, yielding the estimates $\hat{\boldsymbol{\alpha}}_{1}$ and $\hat{\boldsymbol{\alpha}}_{2}$. The final function to maximize is

$$
l\left(\boldsymbol{\theta}_{t}\right)=\sum_{t=p+1}^{T} \log c\left(F_{1}\left(z_{1 t} ; \hat{\boldsymbol{\alpha}}_{1}\right), F_{2}\left(z_{2 t} ; \hat{\boldsymbol{\alpha}}_{2}\right) ; \boldsymbol{\theta}_{t}\right) .
$$

From this, estimates for the dependence parameter $\boldsymbol{\theta}_{t}$ are obtained. Statistical properties of this estimation procedure are to be found in Chen and Fan (2005), Joe (2005) and Patton (2006a).

\section{Data}

The data consist of observations of spot exchange rates for the U.S. dollar, the Euro, and the Japanese Yen, covering the sample period October 1, 2000 until October 1, 2008, equally-spaced at sixty-minutes. The data set was obtained from Olsen Financial Technologies GmbH. The sixty-minute prices are obtained by linear interpolation between the average of the bid and ask tick quotes immediately before and immediately after the sixty-minute time stamps across the 24-hour day.

We compute the returns from prices of 1 U.S. dollar expressed in Euro and Yen, this is, EUR/USD and JPY/USD. Sixty-minute returns are computed 
as the first difference of the logarithm of the sixty-minute prices. Analogously, from the corresponding frequency equally-spaced logarithmic prices, we compute two, four, eight, twelve hours and one day returns, yielding twelve series in all.

High-frequency data is subject to market microstructure frictions. By choosing sixty-minute prices as the highest frequency we achieve a compromise between estimation bias originating in high-frequency bid/ask bounce and variance which increases as the sampling frequency decreases.

We exclude all the returns from Friday 21:00 GMT until Sunday 21:00 GMT and Bank Holidays in order to simplify the inference and avoid modeling weekend and Bank Holiday effects. After deleting these slow trading activity days the data set consists of returns spanning 2,016 days in total.

It is known that seasonal components, associated with working hours of exchanges worldwide, distort the estimation of volatility models, in particular GARCH type models as we use here. ${ }^{5}$ To overcome this problem we compute the intraday seasonal pattern and use it to deseasonalize the sixty-minute returns. We compute a seasonal factor corresponding to each sixty-minute interval of the day as the average of the squared returns observed every day

\footnotetext{
${ }^{5}$ See for instance Martens et al. (2002).
} 
for that time interval,

$$
s_{i}^{2}=\frac{1}{T} \sum_{t=1}^{T} \tilde{x}_{i t}^{2} \quad i=1,2, \ldots, 24,
$$

where $T$ is the number of days in the sample and $\tilde{x}_{i t}$ is the raw return in the $i$ th interval of day $t$. The sixty-minute deseasonalized returns are computed as

$$
x_{i t}=\frac{\tilde{x}_{i t}}{s_{i}} \quad i=1,2, \ldots, 24 \text { and } t=1,2, \ldots, T .
$$

We compute the deseasonalized returns for the other intraday frequencies similarly to (8), where the corresponding seasonal factor is the sum of the sixty-minute factors in that frequency time interval,

$$
\bar{s}_{i}^{2}=\sum_{t=0}^{24 / \delta-1} s_{i 24 / \delta-t}^{2} \quad i=1, \ldots, \delta
$$

where $\delta=12,6,3,2$ for 2-hour, 4-hour, 8-hour and 12-hour return frequency respectively. When clear from the context we refer to the deseasonalized returns simply as returns.

\section{The EUR/USD and JPY/USD univariate returns at different time horizons}

We first analyze the unconditional distribution of the returns at the several time horizons. The modeling of the univariate dynamics and conditional distribution follows. This section ends with model specification tests. 


\subsection{Univariate modeling}

Tables 1 and 2 contain summary statistics of the returns. Observe that neither exchange rate returns shows a significant trend. The returns' skewness is mostly negative for the various time horizons although not revealing strikingly asymmetric returns. At all time horizons, both series show excess kurtosis higher than zero, corresponding to a normally distributed variable.

From the values obtained for the kurtosis we can see that, for both spot rates, the unconditional univariate distributions are clearly heavy tailed for shorter time horizons and become more thin tailed as the time horizon increases. This feature has been found in the financial econometric literature; see for instance Nekhili et al. (2002) where similar tail behavior was found for the JPY/USD exchange rate returns.

The estimated linear correlation decreases as the time horizon decreases. This feature is usually justified in the literature by the non-synchronicity of trades. The fact that trades are not synchronized causes an increasing downwards correlation bias as the time horizon shortens.

\section{[Insert Table 1 here] \\ [Insert Table 2 here]}

Concerning the conditional distributions, the Ljung-Box test statistics up to the twentieth order indicate serial correlation; see Table 3 . We test for the 
presence of conditional heteroscedasticity using the LM test introduced by Engle (1982). The test statistics obtained reveal the presence of time-varying conditional variance at all the time horizons.

\section{[Insert Table 3 here]}

The results obtained from the tests on the univariate return time series justify, in our discrete-time setting, modeling the time-varying volatility with a GARCH specification assuming Student- $t$ innovations. These model assumptions are checked in Section 4.2 with the appropriate specification tests. Tables 4 and 5 give the order of the models fitted and the corresponding parameter estimates. Note that the $t$-distributions fitted at the two and four hours time horizons may have infinite kurtosis $(\hat{\nu}<4)$ and so the fourth moment does not exist.

[Insert Table 4 here]

[Insert Table 5 here]

From the fitted AR-GARCH model parameters we recover the standardized residuals or filtered returns $\hat{z}_{t}$ for each univariate time series $\left(x_{1}, x_{2}, \ldots, x_{T}\right)$. That is

$$
\hat{z}_{t}=\left(x_{t}-\hat{\mu}_{t}\right) / \hat{\sigma}_{t}, \quad t=1,2, \ldots, T \text {. }
$$




\subsection{Model specification tests}

Once the univariate models are selected and fitted, the dynamics and the goodness-of-fit of our assumed Student- $t$ density need to be checked. In Table 6 we report the p-values of the Ljung-Box test and of the AndersonDarling goodness-of-fit test for a Student- $t$ density.

\section{[Insert Table 6 here]}

The Ljung-Box test for the residuals indicates that there is no autocorrelation at all time horizons except for the one hour EUR/USD and four hour JPY/USD. For the squared values of the residuals we have that the two hour EUR/USD and the one and eight hour JPY/USD time horizons fail the autocorrelation test at a significance level of $5 \%$.

Table 6 also reports the p-values of the Student- $t$ model goodness-offit test for the marginal distributions. The goodness-of-fit of the marginal densities, according to the Anderson-Darling test, are not rejected at all time horizons. The linear correlation between the residuals increases with the time horizons as observed for the returns (Table 2).

\section{The EUR/USD and JPY/USD dependence structure at differ- ent time horizons}

In order to fit the copula models we use the standardized residuals $\left\{\left(\hat{z}_{1 t}, \hat{z}_{2 t}\right)\right.$ : $t=0, \ldots, T\}$ defined in (9) together with the estimated degrees of freedom 
$\hat{\nu}_{i}$ from the marginal AR-GARCH modeling above; see Tables 4 and 5 . These data are used in equation (7) where $F_{i}$ is the Student- $t$ distribution assumed for the GARCH innovations.

The copula families used in modeling the data are: Student- $t$, Frank, Plackett, Gaussian, Gumbel and Clayton; for details on these classes see Joe (1997), Embrechts et al. (2002) and Nelsen (2006). This choice of copula models is partly based on previous analyses, tractability, flexibility and to allow for a fairly broad class with respect to extremal clustering and possible asymmetry. If there is asymmetry in the dependence structure we need a copula able to model this feature. Of particular interest are the the upper and lower tail dependence coefficients, as defined in McNeil et al. (2005) page 209. These range from zero in case of asymptotic independence, to one in case of perfect asymptotic tail dependence. The Clayton copula family has lower tail dependence ranging from asymptotic independence to perfect asymptotic tail dependence. Hence, it is convenient to use the mixture Clayton with the survival Clayton model to investigate the existence and asymmetry of upper and lower tail dependence.

Denoting the Clayton copula family with parameter $\theta>0$ by $C^{\mathrm{Cl}}(\cdot, \cdot ; \theta)$, the mixture copula with vector parameter $\boldsymbol{\theta}$ has a distribution function of the form

$$
C\left(u_{1}, u_{2} ; \boldsymbol{\theta}\right)=\theta_{3} C^{\mathrm{Cl}}\left(u_{1}, u_{2} ; \theta_{1}\right)+\left(1-\theta_{3}\right) C^{\mathrm{Cl}}\left(u_{1}, u_{2} ; \theta_{2}\right) .
$$


The AIC values in Table 8 allow us to rank the several models. The best non-dynamic copula models are the Clayton mixture and the $t$-copula for the one, two and four hour time horizons. For the remaining, longer, time horizons the best non-dynamic models are the Placket and the $t$-copula. The $t$-copula continues being the best when we introduce dynamics in the model parameter. The second best dynamic model at all time horizons is the Gaussian. The dynamics used with each copula parameter are listed in Table 7. Further support for the $t$-based models is to be found in Breymann et al. (2003), Daul et al. (2003), Demarta and McNeil (2005), Rosenberg and Schuermann (2006), Pesaran et al. (2004) and Platen and Heath (2006).

$$
\text { [Insert Table } 7 \text { here] }
$$

For the Clayton mixture model defined in (10), we test the null hypothesis $H_{0}: \theta_{1}=\theta_{2}$ and $\theta_{3}=0.5$ versus the alternative $H_{A}: \theta_{1} \neq \theta_{2}$ and $\theta_{3} \neq 0.5$. A high p-value indicates that a three parameter asymmetric Clayton mixture is not significantly better than its one parameter symmetric version. For the six time horizons, from the one hour to the daily, we obtain as p-values 0.8211 , $0.1166,0.2004,0.8148,0.4208$ and 0.3377 respectively. The p-values obtained strongly favor the symmetric model at all time horizons. The estimated coefficient of tail dependence corresponding to the Clayton parameter for the six time horizons, from the one hour to the daily, are $0.4569,0.4267$, $0.4074,0.4240,0.4099$ and 0.3956 , respectively, around the middle of the tail 
coefficient range $(0,1)$.

It is not surprising that exchange rates show symmetric dependence in the data, given that its definition is always against another currency. This fact makes symmetric copula families to be potentially good models for exchange rates dependence. This is not the case in other financial data, such as stock returns for instance, where downside dependence is often stronger than upside dependence.

In order to compare the relative performance of the dynamic models compared to their non-dynamic versions, we perform a likelihood ratio test for each model and time horizon. The test statistics and p-values obtained are in Table 8.

[Insert Table 8 here]

The Gaussian and the $t$-copula dynamic models outperform their nondynamic versions at all time horizons. The dynamic Frank model is significantly better than the non-dynamic model at all time horizons except for the eight hour one. The dynamic Clayton mixture outperforms at the eight and two hour time horizons. The dynamic Gumbel beats the non-dynamic Gumbel at the eight hour time horizon and the Clayton at one and two hours.

In summary, the Gaussian and the $t$-copula models stand out as the best models consistently across all time horizons and their dynamic versions always add significant modeling power. It is remarkable that at all time 
horizons the Gaussian and $t$-copula models with Fisher dynamics lead to a better AIC than the Gaussian and $t$-copula models with Tse-Tsui dynamics.

The parameter estimates and asymptotic standard errors obtained for all Student- $t$ and Gaussian copula models are reported in Table 9 and Table 10. These include the parameter estimates for the corresponding non-dynamic model.

[Insert Table 9 here]

[Insert Table 10 here]

The estimate for $\alpha$ in the $t$-copula model with Fisher dynamics (3) can be considered to be zero for two hours, twelve hours and daily returns. But $\beta$ and $\gamma$ are definitely different from zero for both Gaussian and $t$-copula models with Fisher and Tse-Tsui dynamics, at all time horizons. In other words, the estimated correlation depends on the marginal returns and on the correlation from the previous time period.

The degrees of freedom estimated for the dependence structure is always larger for the dynamic Student- $t$ copula models than for the static $t$-copula model, as can be seen from a comparison of the values listed in Table 9 . Hence, a non-dynamic modeling might induce a spurious heavier conditional tail.

In contrast to multivariate GARCH models, with Gaussian or multivariate Student- $t$ conditional distribution, copula-GARCH models allow us com- 
plete freedom to choose different conditional marginal distributions. In the case of the fitted Student- $t$ copula models we compare the degrees of freedom estimated for each marginal conditional distribution (see Tables 4 and 5) and for the copula (see Table 9). For the daily time horizon, for instance, we estimate $\hat{\nu}=15.8$ for the EUR/USD, $\hat{\nu}=9.2$ for the JPY/USD and $\hat{\nu}=8.4$ for the $t$-copula with Fisher dynamics. Although for our data we cannot conclude that the estimates are statistically different for most of the time horizons, using three parameters for the degrees of freedom must surely contribute to the excellence of the results in the specification tests.

For comparison with the copula-GARCH model we estimate the BEKK model introduced by Engle and Kroner (1995). We model the conditional mean of each return series using the same AR specification as in the marginal modeling in Section 4. Then we estimate a bivariate BEKK model on the residuals. We fit a BEKK model at each of the six time horizons assuming (bivariate) Student- $t$ innovations.

Table 11 lists the corresponding autocorrelation and goodness-of-fit tests. All time horizons pass the autocorrelation test except the one hour time horizon residuals. The one, two and eight hour squared residuals fail the same test for both rates at the $5 \%$ level. Only the JPY/USD residuals for time horizons four, eight and twelve hours pass the goodness-of-fit test for a Student- $t$ distribution. The other nine series fail the test. 
[Insert Table 11 here]

The goodness-of-fit results for the distribution of the residuals are substantially worse than for the copula-GARCH model (see Anderson-Darling test results in Tables 6 and 11). The p-values for testing the Student- $t$ distribution are higher for the copula-GARCH model in ten out of twelve series. The failure of the Student- $t$ distribution might be due to the asymmetry revealed by the estimated skewness (higher than for the original returns) especially for the JPY/USD residuals; see Table 12. The existence of excess kurtosis in the AR-BEKK residuals is consistent with a heavy tailed Student- $t$ distribution.

[Insert Table 12 here]

We conclude that the copula-GARCH model is better specified than the flexible BEKK model.

\section{Evaluating and comparing the correlation estimates at different time horizons}

From the estimated parameters for the dynamic correlation models we compute the time-varying estimated correlation for the Gaussian and Student$t$ copula with Fisher dynamics (3) and Tse-Tsui dynamics (4), and for the BEKK model for all time horizons. As a benchmark we use two nonparametric measures of dependence: Kendall's tau coefficient and realized correlation. 
For all time horizons we compute rolling window estimates of Kendall's tau using a window size of 40 observations. Kendall's tau at day $t$ is computed using the previous 39 pairs of EUR/USD and JPY/USD returns, and the return pair at $t$ itself. Since for our data the best performing copula models belong to the elliptical family we transform Kendall's tau estimates, $\tau_{\text {Kendall }}$, into linear correlation, $\rho_{\tau}$, using the relationship

$$
\tau_{\text {Kendall }, t}=\frac{2}{\pi} \arcsin \rho_{\tau, t},
$$

which is valid for elliptical distributions; see Lindskog et al. (2003). The linear correlation estimated from Kendall's tau is plotted in Figure 1 together with the dynamic Gaussian and Student- $t$ copula with Fisher dynamics (3) and daily frequency. Recall from Table 2 in Section 4 that the unconditional constant correlation estimate is 0.43 . In the period $2007-2008$ we can observe a significant drop in the correlation starting in the beginning of 2007 , bottoming out in the third quarter with a consequent rise reaching the previous levels during the second quarter of 2008. Events having different effects in European and Japanese economies cause changes in the correlation between the two exchange rates. A delay in the effect of the sub-prime crisis on the Asian economies compared with the European can justify the decrease in the correlation observed until mid-2007. After mid-2007 all FX markets, including Euro and Japanese Yen, had suffered equal contagion from the crisis and correlation between Euro and Yen returned to pre-crisis levels. 
[Insert Figure 1 here]

Given these results we can infer strongly that the conditional dependence is time-varying and, consequently, that its dynamics should be modeled. Clearly, assuming that the correlation is constant may cause substantial mispricing and errors in risk measurement.

In order to formally evaluate and benchmark our nonlinear model correlation estimates we follow Andersen et al. (2003). First, we evaluate the correlation estimates obtained from the different models using the Kendall's tau estimates. We perform the regression

$$
\rho_{\tau, t}=b_{0}+b_{1} \rho_{\text {model }, t}+u_{t},
$$

to project the correlation, $\rho_{\tau, t}$, obtained from Kendall's tau using (11) onto a constant and the various parametric model estimates, $\rho_{\text {model }, t}$.

We plot in Figure 2 the regression of the estimates of Kendall's tau for the daily filtered returns, computed according to (11), on the Tse-Tsui dynamics, Fisher dynamics and BEKK model correlation estimates. From these plots we can anticipate superior results for the Fisher dynamics with Gauss and Student- $t$ copula in the regression analysis. The regression of Kendall's tau transformed correlation on the Tse-Tsui dynamics and BEKK model estimates are visibly weaker.

[Insert Figure 2 here] 
We report in Table 13 the numerical results from the evaluation regressions. The results are consistent across models and time horizons. For all models the regression $R^{2}$ is highest for the four hours time horizon. For the four, eight, twelve hour and daily time horizons the two models with Fisher dynamics (Gaussian and $t$-copula) always have the highest $R^{2}$. For the two hour time horizon the two dynamic Gaussian copula models have the highest $R^{2}$. Finally the best $R^{2}$ for the one hour time horizon is for the Gaussian model with Tse-Tsui dynamics and for the BEKK model.

[Insert Table 13 here]

We further evaluate the correlation estimates from a different point of view. We aggregate the model correlation estimates from the different time horizons into daily and compare them with the daily realized correlation and Kendall's tau estimates. The day- $t$ realized correlation is computed from the hourly time horizon returns as

$$
\rho_{\mathrm{RC}, t}=\frac{\sum_{i=1}^{24} x_{i t} \cdot y_{i t}}{\sqrt{\sum_{i=1}^{24} x_{i t}^{2} \sum_{i=1}^{24} y_{i t}^{2}}}, \quad t=1,2, \ldots, T,
$$

where $x_{i t}$ is the hour $i$ EUR/USD return of day $t$, and $y_{i t}$ the JPY/USD return for the same hour and day. With the model correlation estimates at time horizon $h$, for $h=1,2,4,8,12$ hours, we compute the day- $t$ correlation 
estimate as

$$
\rho_{\text {model }, t, h}=\frac{\sum_{i=1}^{24 / h} \rho_{i, t, h} \sigma_{\mathrm{EUR}, i, t, h} \sigma_{\mathrm{JPY}, i, t, h}}{\sqrt{\sum_{i=1}^{24 / h} \sigma_{\mathrm{EUR}, i, t, h}^{2} \sum_{i=1}^{24 / h} \sigma_{\mathrm{JPY}, i, t, h}^{2}}}, \quad t=1,2, \ldots, T,
$$

where $\rho_{i, t, h}$ is the time horizon $h$ estimated correlation for time period $i$ of day $t$. For instance, $\rho_{3, t, 2}$ is the two hour time horizon correlation estimated for 4-6am of day $t$. The $\sigma_{\mathrm{EUR}, i, t, h}^{2}$ and $\sigma_{\mathrm{JPY}, i, t, h}^{2}$ are the marginal variances for the same time period, obtained from the univariate time series modeling.

In order to compare the correlation estimates obtained from the different time horizons we use the regression

$$
\rho_{\mathrm{RC}, t}=b_{0}+b_{1} \rho_{\text {model }, t, h}+u_{t} .
$$

to project the realized correlation $\rho_{\mathrm{RC}, t}$ on a constant and on the model estimates $\rho_{\text {model }, t, h}$. We also regress the daily Kendall's tau correlation, $\rho_{\tau, t}$, on the daily model correlations obtained from the different time horizons as in (12). The results are reported in Table 14 .

[Insert Table 14 here]

The $R^{2}$ for the realized correlation increases with the time frequency used to compute the daily correlations, and this consistently for all the models. The shorter the time horizon the higher the $R^{2}$. For each time horizon the BEKK model has the higher $R^{2}$. The $t$ and Gaussian models with Fisher dynamics are the second best. Only for the one hour time horizon the second best $R^{2}$ 
model is the Gaussian with Tse-Tsui dynamics. The conclusion here is that, when measured against the realized correlation, using shorter time horizons improves the dynamic daily correlation estimates.

When regressing the daily rolling window Kendall's tau correlation on the daily correlations estimated from different time horizons, the results (see Table 14) are still consistent across time horizons and models. The $R^{2}$ is higher when the correlations are estimated from longer time horizons. The highest $R^{2}$ is obtained from the $t$ model with Fisher dynamics for all frequencies except one and eight hours where the Gaussian model with Fisher dynamics is better. The BEKK model has the lowest $R^{2}$ for all time horizons except for the eight hours where it ranks the second to last. When measured against the Kendall's tau correlation the results show no advantage in using shorter time horizons for modeling the daily correlation. Across models the Fisher dynamics perform consistently better than the Tse-Tsui dynamics and the BEKK model.

Implications for the choice of sampling frequency resulting from regressing the realized correlation are different to those resulting from regressing Kendall's tau. This may be due to the different conceptual nature of the benchmark models. 


\section{Conclusion}

The contribution of the paper is threefold. First of all, we want to contribute to the ongoing discussion between practitioners and academics in order to advance the methodological basis for risk measurement technology. There is a need to move beyond linear correlation and, through the notion of copula, our paper contributes to this goal. Second, we propose a parsimonious conditional dependence model, the copula with Fisher dynamics model, taking dynamics in the dependence structure into account and use it to analyze the dependence between Euro and Japanese Yen. Thirdly we show the superiority of the proposed model compared to models previously investigated in the literature.

Looking first at the univariate conditional distributions of the EUR/USD and JPY/USD returns we conclude they are well described by a Student- $t$ distribution and this from hourly up to daily return frequencies. We observe some tendency for the tail of the distribution to become heavier as the time frequency increases.

To model dependence, comparing different copula models, the $t$-copula performs best for the time-invariant models and the Gaussian and $t$-copula models are the best for the time-varying models. Combining different copula families and dynamic specifications we conclude that the dependence between the EUR/USD and JPY/USD is time-varying. The best time-varying models 
(Gaussian and $t$-copula) are statistically better than the corresponding timeinvariant model. All these results are obtained consistently at all the time horizons considered.

Testing for different upper and lower tail dependence revealed no asymmetry, a natural feature of FX data.

We observe that the degrees of freedom of each Student- $t$ margin and copula are different at all the time horizons considered. We find a heavier tail in the marginal exchange rates than in the dependence structure. Although not always statistically significant, this observation raises concerns about using a bivariate Student- $t$ for the conditional distribution of the returns.

We find that the degrees of freedom estimated for the dynamic $t$-copula model are significantly higher than for the time-invariant model. The timeinvariant model seems to overestimate the dependence tail heaviness.

The dependence paths estimated using the time-varying copula-GARCH models have very high significance at all time horizons when compared to two non-parametric measures of dependence: realized correlation and Kendall's tau.

We found a remarkable decrease in the dependence between the EUR/USD and JPY/USD returns during the first three quarters of 2007 followed by an increase till mid 2008 back to values observed before 2007. This decline in the correlation was possibly the result of a delay in the effects of the sub-prime 
crisis on the Japanese Yen compared with the effect on the Euro. Once both currencies became equally influenced by the crisis their dependence went back to pre-crisis values.

We conclude that the proposed copula with Fisher transformation dynamics model is superior to alternative models previously tested in the literature and that its use should be considered in preference to these models in future work.

\section{Acknowledgments}

We acknowledge useful discussions with Alexander McNeil, Alessandro Palandri, Gordon Gemmill, Mark Salmon, Nick Webber and anonymous referees for detailed comments on earlier versions of this paper. 


\section{References}

Andersen, T. G., Bollerslev, T., Diebold, F. X., Labys, P., 2003. Modeling and forecasting realized volatility. Econometrica 71, 579-625.

Andreou, E., Ghysels, E., 2003. Tests for breaks in the conditional comovements of assets returns. Statistica Sinica 13, 1045-73.

Bauwens, L., Laurent, S., Rombouts, J. V. K., 2006. Multivariate GARCH models: a survey. Journal of Applied Econometrics 21 (1), 79-109.

Bollerslev, T., Engle, R. F., Wooldridge, J. M., 1988. A capital asset pricing model with time-varying covariances. Journal of Political Economy 96, $116-31$.

Boyer, B. H., Gibson, M. S., Loretan, M., 1999. Pitfalls in tests for changes in correlations. International finance discussion paper No. 597, Board of Governors of the Federal Reserve System.

Breymann, W., Dias, A., Embrechts, P., 2003. Dependence structures for multivariate high-frequency data in finance. Quantitative Finance 3, 1-14.

Chen, X., Fan, Y., 2005. Estimation of copula-based semiparametric time series models. Journal of Econometrics 130 (2), 307-35.

Daul, S., Giorgi, E. D., Lindskog, F., McNeil, A., 2003. Using the grouped t-copula. RISK Magazine, 73-6.

Demarta, S., McNeil, A. J., 2005. The t copula and related copulas. International Statistical Review 73 (1), 111-29.

Drost, F. C., Werker, B. J. M., 1996. Closing the GARCH gap: Continuous time GARCH modeling. Journal of Econometrics 74, 31-57. 
Embrechts, P., McNeil, A. J., Straumann, D., 2002. Correlation and dependence in Risk Management: Properties and pitfalls, in: Dempster, M. (Eds.), Risk Management: Value at Risk and Beyond, Cambridge University Press, Cambridge, pp. 176-223.

Engle, R., 2009. Anticipating Correlations: A New Paradigm for Risk Management. Princeton University Press, Princeton, NJ.

Engle, R., Sheppard, K., 2001. Theoretical and empirical properties of dynamic conditional correlation MVGARCH. Working Paper No. 2001-15, University of California, San Diego.

Engle, R. F., 1982. Autoregressive conditional heteroscedasticity with estimates of the variance of United Kingdom inflation. Econometrica 50 (4), 987-1007.

Engle, R. F., Kroner, K. F., 1995. Multivariate simultaneous generalized ARCH. Econometric Theory 11 (1), 122-50.

Fortin, I., Kuzmics, C., 2002. Tail-dependence in stock return-pairs. International Journal of Intelligent Systems in Accounting, Finance \& Management $11(2), 89-107$.

Hamilton, J. D., 1990. Analysis of time series subject to changes in regime. Journal of Econometrics 45 (1-2), 39-70.

Joe, H., 1997. Multivariate Models and Dependence Concepts. Chapman \& Hall, London.

Joe, H., 2005. Asympotic efficiency of the two-stage estimation method for copula-based models. Journal of Multivariate Analysis 94, 401-19. 
Jondeau, E., Rockinger, M., 2006. The copula-GARCH model of conditional dependencies: An international stock-market application. Journal of International Money and Finance 25 (5), 827-53.

Lindskog, F., McNeil, A. J., Schmock, U., 2003. Kendall's tau for elliptical distributions. In: Credit Risk-Measurement, Evaluation and Management (Ed. G. Bol, G. Nakhaeizadeh, S. T. Rachev, T. Ridder and K.-H. Vollmer), Physica-Verlag, Heidelberg, 149-56.

Longin, F., Solnik, B., 2001. Extreme correlation of international equity markets. Journal of Finance LVI (2), 649-76.

Loretan, M., English, W. B., 2000. Evaluating correlation breakdowns during periods of market volatility. International Finance Working Paper No.658, Board of Governors of the Federal Reserve System.

Loretan, M., Phillips, P. C. B., 1994. Testing the covariance stationarity of heavy-tailed time series: An overview of the theory with applications to several financial data sets. Journal of Empirical Finance 1 (2), 211-48.

Martens, M., Chang, Y. C., Taylor, S. J., 2002. A comparison of seasonal adjustment methods when forcasting intraday volatility. Journal of Financial Research 25, 283-99.

McNeil, A. J., Frey, R., Embrechts, P., 2005. Quantitative Risk Management: Concepts, Techniques and Tools. Princeton University Press, Princeton, NJ.

Meddahi, N., Renault, E., 2004. Temporal aggregation of volatility models. Journal of Econometrics 119, 355-79.

Nekhili, R., Altay-Salih, A., Gençay, R., 2002. Exploring exchange rate returns at different time horizons. Physica A 313, 671-82. 
Nelsen, R. B., 2006. An introduction to copulas, 2nd Edition. Springer Series in Statistics. Springer, New York.

Patton, A. J., 2006a. Estimation of multivariate models for time series of possibly different lengths. Journal of Applied Econometrics 21, 147-73.

Patton, A. J., 2006b. Modelling asymmetric exchange rate dependence. International Economic Review 47 (2), 527-56.

Pesaran, M. H., Schuermann, T., Weiner, S. M., 2004. Modeling regional interdependencies using a global error-correcting macroeconometric model. Journal of Business \& Economic Statistics 22 (2), 129-62.

Platen, E., Heath, D., 2006. A Benchmark Approach to Quantitative Finance. Springer Finance, Springer, Berlin.

Rosenberg, J. V., Schuermann, T., 2006. A general approach to integrated risk management with skewed, fat-tailed risks. Journal of Financial Economics 79 (3), 569-614.

Sklar, A., 1959. Fonctions de répartition à $n$ dimensions et leurs marges. Publications de l'Institut de Statistique de L'Université de Paris 8, 22931.

Tse, Y. K., Tsui, A. K. C., 2002. A multivariate generalized autorregressive conditional heteroscedasticity model with time-varying correlations. Journal of Business \& Economic Statistics 20 (3), 351-62. 
Table 1: Summary statistics: mean and standard deviation

\begin{tabular}{lrcrrr}
\hline & \multicolumn{2}{c}{ EUR/USD } & & \multicolumn{2}{c}{ JPY/USD } \\
\cline { 2 - 3 } \cline { 5 - 6 } Time horizon & Mean & Std. dev. & & Mean & Std. dev. \\
\hline 1 hour & -0.0143 & 0.9999 & & -0.0007 & 1.0000 \\
2 hours & -0.0154 & 1.0131 & & -0.0005 & 1.0147 \\
4 hours & -0.0175 & 1.0181 & & 0.0032 & 0.9926 \\
8 hours & -0.0271 & 0.9970 & & -0.0064 & 0.9714 \\
12 hours & -0.0185 & 0.9476 & & 0.0029 & 0.9546 \\
1 day & -0.0459 & 1.0212 & & 0.0061 & 0.9903 \\
\hline
\end{tabular}

Notes: Sample mean and standard deviation of the EUR/USD and JPY/USD returns at the six time horizons. 
Table 2: Summary statistics: skewness, kurtosis, and correlation

\begin{tabular}{|c|c|c|c|c|c|}
\hline \multirow[b]{2}{*}{ Time horizon } & \multicolumn{2}{|c|}{ EUR/USD } & \multicolumn{2}{|c|}{ JPY/USD } & \multirow{2}{*}{$\begin{array}{c}\text { Linear } \\
\text { correlation }\end{array}$} \\
\hline & Skewness & Kurtosis & Skewness & Kurtosis & \\
\hline 1 hour & -0.0624 & 6.854 & -0.0838 & 7.493 & 0.3878 \\
\hline 2 hours & 0.0044 & 5.287 & -0.0149 & 4.876 & 0.4040 \\
\hline 4 hours & -0.3074 & 7.391 & -0.0641 & 3.066 & 0.4030 \\
\hline 8 hours & -0.0430 & 2.978 & -0.1743 & 2.349 & 0.4088 \\
\hline 12 hours & -0.0433 & 2.244 & -0.1211 & 2.080 & 0.4016 \\
\hline 1 day & 0.0703 & 0.711 & -0.0967 & 1.070 & 0.4311 \\
\hline
\end{tabular}

Notes: Sample skewness, excess kurtosis and linear correlation of the EUR/USD and JPY/USD returns at the six time horizons. 
Table 3: Ljung-Box (L-B) and ARCH LM (LM) tests

\begin{tabular}{|c|c|c|c|c|c|c|c|c|}
\hline \multirow[b]{2}{*}{ Time horizon } & \multicolumn{4}{|c|}{ EUR/USD } & \multicolumn{4}{|c|}{ JPY/USD } \\
\hline & L-B & $\mathrm{p}$-value & $\mathrm{LM}$ & p-value & L-B & $\mathrm{p}$-value & $\mathrm{LM}$ & $\mathrm{p}$-value \\
\hline 1 hour & 45.74 & 0.00 & 1143.90 & 0.00 & 40.13 & 0.00 & 2093.60 & 0.00 \\
\hline 2 hours & 48.04 & 0.00 & 556.70 & 0.00 & 55.30 & 0.00 & 820.54 & 0.00 \\
\hline 4 hours & 33.14 & 0.03 & 165.46 & 0.00 & 56.78 & 0.00 & 439.64 & 0.00 \\
\hline 8 hours & 35.53 & 0.01 & 172.03 & 0.00 & 33.95 & 0.02 & 257.78 & 0.00 \\
\hline 12 hours & 34.91 & 0.02 & 130.37 & 0.00 & 34.46 & 0.03 & 206.58 & 0.00 \\
\hline 1 day & 26.71 & 0.14 & 126.94 & 0.00 & 30.85 & 0.06 & 75.47 & 0.00 \\
\hline
\end{tabular}

Notes: Ljung-Box (L-B) and ARCH LM (LM) test statistics up to the twentieth order for the EUR/USD and JPY/USD returns at the six time horizons. The null hypothesis of no serial correlation in returns is rejected at $5 \%$ level for all time horizons except for the daily. The null hypothesis of no ARCH effects is rejected for all time horizons at the $5 \%$ level. 
Table 4: Results from the univariate modeling of EUR/USD

\begin{tabular}{lcccccc}
\hline Time horizon & 1 day & 12 hours & 8 hours & 4 hours & 2 hours & 1 hour \\
\hline Constant, $\hat{\mu}$ & -0.0476 & & & -0.0116 & -0.0153 & -0.0119 \\
& $(0.0209)$ & & & $(0.0068)$ & $(0.0047)$ & $(0.0033)$ \\
AR $(1), \hat{\phi}_{1}$ & & - & - & -0.0220 & - & - \\
& & & & $(0.0079)$ & & \\
AR $(2), \hat{\phi}_{2}$ & & - & - & 0.0165 & - & - \\
& & & & $(0.0076)$ & & \\
GARCH constant, $\hat{\alpha}_{0}$ & - & - & 0.0013 & 0.0197 & 0.0007 & 0.0007 \\
& & & $(0.0058)$ & $(0.0006)$ & $(0.0003)$ & $(0.0002)$ \\
Lag $1 \epsilon^{2}, \hat{\alpha}_{1}$ & - & 0.0232 & - & 0.0196 & 0.0162 & 0.0753 \\
& $(0.038)$ & & $(0.0026)$ & $(0.0020)$ & $(0.0098)$ \\
Lag $2 \epsilon^{2}, \hat{\alpha}_{2}$ & 0.0587 & - & 0.0074 & - & - & -0.0623 \\
& $(0.0188)$ & & $(0.0028)$ & & & $(0.0082)$ \\
Lag 1 variance, $\hat{\beta}_{1}$ & 0.9617 & 0.9763 & 0.9664 & 0.9813 & 0.9844 & 0.9866 \\
& $(0.0071)$ & $(0.0037)$ & $(0.0078)$ & $(0.0025)$ & $(0.0021)$ & $(0.0022)$ \\
Degrees of freedom, $\hat{\nu}$ & 15.8061 & 5.4046 & 5.1034 & 3.7330 & 3.7480 & 4.1117 \\
& $(4.1700)$ & $(0.4550)$ & $(0.3284)$ & $(0.1266)$ & $(0.0881)$ & $(0.0713)$ \\
\hline
\end{tabular}

Notes: Maximum likelihood estimates and corresponding asymptotic standard errors (in parentheses) obtained from fitting AR-GARCH models to the return series EUR/USD at each time horizon. 
Table 5: Results from the univariate modeling of JPY/USD

\begin{tabular}{|c|c|c|c|c|c|c|}
\hline Time horizon & 1 day & 12 hours & 8 hours & 4 hours & 2 hours & 1 hour \\
\hline Constant, $\hat{\mu}$ & - & - & - & $\begin{array}{c}0.0169 \\
(0.0072)\end{array}$ & $\begin{array}{c}0.0111 \\
(0.0049)\end{array}$ & $\begin{array}{c}0.0078 \\
(0.0034)\end{array}$ \\
\hline $\operatorname{AR}(1), \hat{\phi}_{1}$ & - & - & - & - & $\begin{array}{c}-0.0533 \\
(0.0061)\end{array}$ & - \\
\hline GARCH constant, $\hat{\alpha}_{0}$ & $\begin{array}{c}0.0280 \\
(0.0102)\end{array}$ & $\begin{array}{c}0.0088 \\
(0.0036)\end{array}$ & - & $\begin{array}{c}0.0079 \\
(0.0031)\end{array}$ & $\begin{array}{c}0.0045 \\
(0.0016)\end{array}$ & $\begin{array}{c}0.0092 \\
(0.0025)\end{array}$ \\
\hline $\operatorname{Lag} 1 \epsilon^{2}, \hat{\alpha}_{1}$ & $\begin{array}{c}0.0624 \\
(0.0103)\end{array}$ & - & $\begin{array}{c}0.0031 \\
(0.0004)\end{array}$ & $\begin{array}{c}0.0347 \\
(0.0055)\end{array}$ & $\begin{array}{c}0.0796 \\
(0.0106)\end{array}$ & $\begin{array}{c}0.0356 \\
(0.0051)\end{array}$ \\
\hline $\operatorname{Lag} 2 \epsilon^{2}, \hat{\alpha}_{2}$ & - & $\begin{array}{c}0.0517 \\
(0.0134)\end{array}$ & - & - & $\begin{array}{l}-0.0548 \\
(0.0105)\end{array}$ & - \\
\hline Lag 1 variance, $\hat{\beta}_{1}$ & $\begin{array}{c}0.9108 \\
(0.0143)\end{array}$ & $\begin{array}{c}0.9480 \\
(0.0085)\end{array}$ & $\begin{array}{c}0.9647 \\
(0.0051)\end{array}$ & $\begin{array}{c}0.9600 \\
(0.0076)\end{array}$ & $\begin{array}{c}0.9731 \\
(0.0049)\end{array}$ & $\begin{array}{c}0.9568 \\
(0.0072)\end{array}$ \\
\hline Degrees of freedom, $\hat{\nu}$ & $\begin{array}{c}9.2553 \\
(1.6769)\end{array}$ & $\begin{array}{c}5.8374 \\
(0.4944)\end{array}$ & $\begin{array}{c}4.3450 \\
(0.2921)\end{array}$ & $\begin{array}{c}4.4333 \\
(0.1794)\end{array}$ & $\begin{array}{c}3.7322 \\
(0.0854)\end{array}$ & $\begin{array}{c}4.0833 \\
(0.0749)\end{array}$ \\
\hline
\end{tabular}

Notes: Maximum likelihood estimates and corresponding asymptotic standard errors (in parentheses) obtained from fitting AR-GARCH models to the return series JPY/USD at each time horizon. 
Table 6: Properties of the copula-GARCH model residuals (filtered returns)

\begin{tabular}{|c|c|c|c|c|c|c|c|}
\hline \multirow{2}{*}{$\begin{array}{l}\text { Time } \\
\text { horizon }\end{array}$} & \multicolumn{2}{|c|}{ EUR/USD } & \multicolumn{2}{|c|}{ JPY/USD } & \multicolumn{2}{|c|}{ A-D test } & \multirow{2}{*}{$\begin{array}{c}\text { Linear } \\
\text { correlation }\end{array}$} \\
\hline & $z_{t}$ & $z_{t}^{2}$ & $z_{t}$ & $z_{t}^{2}$ & $\mathrm{EUR} / \$$ & $\mathrm{JPY} / \$$ & \\
\hline 1 hour & 0.0063 & 0.1740 & 0.1361 & 0.0001 & 0.3242 & 0.1327 & 0.4198 \\
\hline 2 hours & 0.0489 & 0.0002 & 0.0479 & 0.2290 & 0.4471 & 0.1501 & 0.4298 \\
\hline 4 hours & 0.2085 & 0.2680 & 0.0016 & 0.2090 & 0.1069 & 0.1704 & 0.4244 \\
\hline 8 hours & 0.3894 & 0.8379 & 0.1257 & 0.0001 & 0.3044 & 0.1137 & 0.4091 \\
\hline 12 hours & 0.2622 & 0.1479 & 0.3806 & 0.4281 & 0.1105 & 0.5220 & 0.4253 \\
\hline 1 day & 0.7408 & 0.1560 & 0.1145 & 0.5418 & 0.6912 & 0.9918 & 0.4498 \\
\hline
\end{tabular}

Notes: The first left four columns have the Ljung-Box autocorrelation test p-values up to the order twelve for the EUR/USD and JPY/USD residuals, $z_{t}$, and squared residuals, $z_{t}^{2}$, at the six time horizons. Columns five and six have the p-values from the Anderson-Darling (A-D) goodness of fit test. The null hypothesis is that the residuals follow a Student- $t$ distribution. The right column displays the linear correlation between the EUR/USD and JPY/USD residuals. 
Table 7: Dynamic copula parameter specification

\begin{tabular}{|c|c|c|}
\hline Copula model & Parameter specification & \\
\hline Clayton & $\theta_{t}=\alpha+\beta\left|z_{1 t-1} z_{2 t-1}\right|+\gamma \theta_{t-1}$ & $\theta_{t}>0$ \\
\hline Frank & $\theta_{t}=\alpha+\beta\left|z_{1 t-1} z_{2 t-1}\right|+\gamma \theta_{t-1}$ & $\theta_{t} \in \mathbb{R} \backslash\{0\}$ \\
\hline Gauss/t Fisher & $\begin{array}{c}\rho_{t}=h^{-1}\left(\alpha+\beta \operatorname{sign}\left(z_{1 t-1} z_{2 t-1}\right) \sqrt{\left|z_{1 t-1} z_{2 t-1}\right|}+\gamma h\left(\rho_{t-1}\right)\right) \\
h(\rho)=\log ((1+\rho) /(1-\rho))\end{array}$ & $-1 \leq \rho_{t} \leq 1$ \\
\hline Gauss/ $t$ Tse-Tsui & $\begin{aligned} \rho_{t}= & (1-\beta-\gamma) \rho+\beta \xi_{t-1}+\gamma \rho_{t-1} \\
& \xi_{t-1}=\sum_{h=1}^{2} z_{1 t-h} z_{2 t-h} / \sqrt{\sum_{h=1}^{2} z_{1 t-h}^{2} \sum_{h=1}^{2} z_{2 t-h}^{2}}\end{aligned}$ & $\begin{array}{l}-1 \leq \rho_{t}, \rho \leq 1 \\
\beta+\gamma<1\end{array}$ \\
\hline Gumbel & $\theta_{t}=\alpha+\beta\left|z_{1 t-1} z_{2 t-1}\right|+\gamma \theta_{t-1}$ & $\theta_{t} \geq 1$ \\
\hline Plackett & $\theta_{t}=(1-\alpha-\beta) \theta+\alpha\left|z_{1 t-1} z_{2 t-1}\right|+\beta \theta_{t-1}$ & $\theta_{t}, \theta \geq 0$ \\
\hline
\end{tabular}

Notes: Parameter dynamics assumed for the different copula models used on modeling the EUR/USD and JPY/USD returns. 
Table 8: Bivariate residuals modeling

\begin{tabular}{|c|c|c|c|c|c|}
\hline \multirow{2}{*}{$\begin{array}{c}\text { Time } \\
\text { horizon }\end{array}$} & \multirow{2}{*}{$\begin{array}{l}\text { Bivariate } \\
\text { model }\end{array}$} & \multicolumn{2}{|c|}{ AIC } & \multicolumn{2}{|c|}{ Log-likelihood ratio } \\
\hline & & static & dynamic & statistic & p-value \\
\hline \multirow{9}{*}{1 hour } & Clayton & -7771.42 & -9246.01 & 1478.59 & 0.00 \\
\hline & Frank & -8687.40 & -10407.05 & 1723.65 & 0.00 \\
\hline & Gaussian Fisher & -9098.03 & -12647.45 & 3553.42 & 0.00 \\
\hline & Gaussian Tse-Tsui & -9098.03 & -11937.74 & 2841.71 & 0.00 \\
\hline & Gumbel & -9502.63 & -9400.70 & -99.92 & 1.00 \\
\hline & Clay \& s.Clay & -10246.42 & -5916.07 & -4328.35 & 1.00 \\
\hline & Plackett & -9514.47 & -9513.53 & 1.06 & 0.30 \\
\hline & $t$ Fisher & -11200.68 & -13972.26 & 2775.59 & 0.00 \\
\hline & $t$ Tse-Tsui & -11200.68 & -13895.25 & 2698.57 & 0.00 \\
\hline \multirow{9}{*}{2 hours } & Clayton & -4187.39 & -4945.23 & 761.84 & 0.00 \\
\hline & Frank & -4577.34 & -5543.39 & 970.04 & 0.00 \\
\hline & Gaussian Fisher & -4798.44 & -6758.40 & 1963.96 & 0.00 \\
\hline & Gaussian Tse-Tsui & -4798.44 & -6434.96 & 1638.52 & 0.00 \\
\hline & Gumbel & -5088.21 & -4841.50 & -242.70 & 1.00 \\
\hline & Clay \& s.Clay & -5453.88 & -6374.41 & 922.53 & 0.00 \\
\hline & Plackett & -5102.69 & -5100.50 & -0.19 & 1.00 \\
\hline & $t$ Fisher & -6084.28 & -7585.54 & 1505.27 & 0.00 \\
\hline & $t$ Tse-Tsui & -6084.28 & -7554.79 & 1474.51 & 0.00 \\
\hline \multirow{9}{*}{4 hours } & Clayton & -2186.47 & -2183.67 & 1.20 & 0.55 \\
\hline & Frank & -2459.60 & -3012.79 & 557.18 & 0.00 \\
\hline & Gaussian Fisher & -2479.79 & -3632.71 & 1156.92 & 0.00 \\
\hline & Gaussian Tse-Tsui & -2479.79 & -3442.02 & 964.23 & 0.00 \\
\hline & Gumbel & -2582.63 & -2554.93 & -23.70 & 1.00 \\
\hline & Clay \& s.Clay & -2787.72 & -2761.99 & -23.72 & 1.00 \\
\hline & Plackett & -2736.84 & -2734.44 & -0.40 & 1.00 \\
\hline & $t$-Fisher & -3105.85 & -3990.58 & 888.73 & 0.00 \\
\hline & $t$ Tse-Tsui & -3105.85 & -3939.56 & 837.71 & 0.00 \\
\hline \multirow{9}{*}{8 hours } & Clayton & -1003.97 & -1006.14 & 6.17 & 0.05 \\
\hline & Frank & -1241.78 & -1242.20 & 4.42 & 0.11 \\
\hline & Gaussian Fisher & -1209.32 & -1709.24 & 503.92 & 0.00 \\
\hline & Gaussian Tse-Tsui & -1209.32 & -1662.27 & 454.95 & 0.00 \\
\hline & Gumbel & -1217.20 & -1220.23 & 7.03 & 0.03 \\
\hline & Clay \& s.Clay & -1280.97 & -1283.22 & 4.25 & 0.04 \\
\hline & Plackett & -1355.58 & -1353.72 & 0.14 & 0.71 \\
\hline & $t$ Fisher & -1422.60 & -1825.00 & 406.40 & 0.00 \\
\hline & $t$ Tse-Tsui & -1422.60 & -1805.93 & 387.32 & 0.00 \\
\hline \multirow{9}{*}{12 hours } & Clayton & -707.36 & -704.68 & 1.32 & 0.52 \\
\hline & Frank & -854.58 & -850.52 & -0.06 & 1.00 \\
\hline & Gaussian Fisher & -842.98 & -1189.56 & 350.58 & 0.00 \\
\hline & Gaussian Tse-Tsui & -842.98 & -1119.11 & 278.13 & 0.00 \\
\hline & Gumbel & -881.40 & -878.38 & 0.98 & 0.61 \\
\hline & Clay \& s.Clay & -922.55 & -918.83 & -1.72 & 1.00 \\
\hline & Plackett & -947.95 & -945.77 & -0.17 & 1.00 \\
\hline & $t$ Fisher & -1029.84 & -1295.02 & 269.18 & 0.00 \\
\hline & $t$ Tse-Tsui & -1029.84 & -1267.28 & 241.44 & 0.00 \\
\hline \multirow{9}{*}{1 day } & Clayton & -390.52 & -387.71 & 1.1886 & 0.55 \\
\hline & Frank & -512.61 & -549.68 & 41.07 & 0.00 \\
\hline & Gaussian Fisher & -475.58 & -600.16 & 128.58 & 0.00 \\
\hline & Gaussian Tse-Tsui & -475.58 & -590.26 & 116.67 & 0.00 \\
\hline & Gumbel & -467.69 & -462.69 & -0.99 & 1.00 \\
\hline & Clay \& s.Clay & -494.47 & -489.77 & -2.70 & 1.00 \\
\hline & Plackett & -546.84 & -543.09 & -1.75 & 1.00 \\
\hline & $t$ Fisher & -538.11 & -633.83 & 99.71 & 0.00 \\
\hline & $t$ Tse-Tsui & -538.11 & -628.47 & 94.35 & 0.00 \\
\hline
\end{tabular}

Notes: Bivariate copula models fitted to the residuals on EUR/USD and JPY/USD logreturns at the six different time horizons. The table lists estimates and asymptotic standard errors of parameters for the static and dynamic copula models. 
Table 9: Estimates for the $t$-copula models

\begin{tabular}{|c|c|c|c|c|c|}
\hline \multirow{2}{*}{$\begin{array}{c}\text { Time } \\
\text { horizon }\end{array}$} & \multicolumn{5}{|c|}{ Parameter Estimates (s.e.) } \\
\hline & & non-dynamic & & $t$ Fisher & $t$ Tse-Tsui \\
\hline \multirow{4}{*}{1 hour } & $\hat{\nu}$ & $4.6369(0.1223)$ & $\hat{\nu}$ & $6.1284(0.2026)$ & $6.0224(0.1964)$ \\
\hline & $\hat{\rho}$ & $0.4209(0.0041)$ & $\hat{\alpha}$ & $0.0005(0.0002)$ & - \\
\hline & & & $\hat{\beta}$ & $0.0338(0.0021)$ & $0.0095(0.0006)$ \\
\hline & & & $\hat{\gamma}$ & $0.9893(0.0007)$ & $0.9908(0.0006)$ \\
\hline \multirow{4}{*}{2 hours } & $\hat{\nu}$ & $4.0779(0.1385)$ & $\hat{\nu}$ & $5.2857(0.2222)$ & $5.1875(0.2151)$ \\
\hline & $\hat{\rho}$ & $0.4312(0.0058)$ & $\hat{\alpha}$ & $0.0008(0.0004)$ & - \\
\hline & & & $\hat{\beta}$ & $0.0447(0.0034)$ & $0.0137(0.0010)$ \\
\hline & & & $\hat{\gamma}$ & $0.9862(0.0011)$ & $0.9860(0.0012)$ \\
\hline \multirow{4}{*}{4 hours } & $\hat{\nu}$ & $4.2469(0.2080)$ & $\hat{\nu}$ & $5.8504(0.3728)$ & $5.6622(0.3530)$ \\
\hline & $\hat{\rho}$ & $0.4450(0.0080)$ & $\hat{\alpha}$ & $0.0030(0.0011)$ & - \\
\hline & & & $\hat{\beta}$ & $0.0746(0.0071)$ & $0.0236(0.0023)$ \\
\hline & & & $\hat{\gamma}$ & $0.9756(0.0027)$ & $0.9735(0.0030)$ \\
\hline \multirow{4}{*}{8 hours } & $\hat{\nu}$ & $5.4654(0.4531)$ & $\hat{\nu}$ & $8.0167(0.9059)$ & $7.9486(0.9093)$ \\
\hline & $\hat{\rho}$ & $0.4431(0.0111)$ & $\hat{\alpha}$ & $0.0087(0.0032)$ & - \\
\hline & & & $\hat{\beta}$ & $0.1042(0.0142)$ & $0.0401(0.0055)$ \\
\hline & & & $\hat{\gamma}$ & $0.9601(0.0066)$ & $0.9458(0.0086)$ \\
\hline \multirow{4}{*}{12 hours } & $\hat{\nu}$ & $4.5832(0.4116)$ & $\hat{\nu}$ & $6.5264(0.7888)$ & $6.088(0.6963)$ \\
\hline & $\hat{\rho}$ & $0.4520(0.0137)$ & $\hat{\alpha}$ & $0.0061(0.0033)$ & - \\
\hline & & & $\hat{\beta}$ & $0.0923(0.0161)$ & $0.0279(0.0050)$ \\
\hline & & & $\hat{\gamma}$ & $0.9663(0.0070)$ & $0.9663(0.0071)$ \\
\hline \multirow{4}{*}{1 day } & $\hat{\nu}$ & $6.1866(0.9520)$ & $\hat{\nu}$ & $8.4611(1.6871)$ & $8.3670(1.6592)$ \\
\hline & $\hat{\rho}$ & $0.4821(0.0178)$ & $\hat{\alpha}$ & $0.0089(0.0056)$ & - \\
\hline & & & $\hat{\beta}$ & $0.0922(0.0190)$ & $0.0400(0.0097)$ \\
\hline & & & $\hat{\gamma}$ & $0.9618(0.0093)$ & $0.9406(0.0168)$ \\
\hline
\end{tabular}

Notes: Parameter estimates and asymptotic standard errors for the three $t$-copula models, non-dynamic, with Fisher transformation dynamic correlation and with TseTsui dynamic correlation, fitted to the hourly up to daily returns on EUR/USD and JPY/USD rates. 
Table 10: Estimates for the Gaussian copula models

\begin{tabular}{|c|c|c|c|c|c|}
\hline \multirow{2}{*}{$\begin{array}{c}\text { Time } \\
\text { horizon }\end{array}$} & \multicolumn{5}{|c|}{ Parameter Estimates (s.e.) } \\
\hline & & non-dynamic & & Gauss Fisher & Gauss Tse-Tsui \\
\hline \multirow{3}{*}{1 hour } & $\hat{\rho}$ & $0.4141(0.0034)$ & $\hat{\alpha}$ & $0.0006(0.0002)$ & - \\
\hline & & & $\hat{\beta}$ & $0.0326(0.0018)$ & $0.0121(0.0008)$ \\
\hline & & & $\hat{\gamma}$ & $0.9895(0.0006)$ & $0.9875(0.0009)$ \\
\hline \multirow{3}{*}{2 hours } & $\hat{\rho}$ & $0.4244(0.0048)$ & $\hat{\alpha}$ & $0.0012(0.0004)$ & - \\
\hline & & & $\hat{\beta}$ & $0.0442(0.0030)$ & $0.0179(0.0013)$ \\
\hline & & & $\hat{\gamma}$ & $0.9859(0.0010)$ & $0.9808(0.0016)$ \\
\hline \multirow{3}{*}{4 hours } & $\hat{\rho}$ & $0.4310(0.0068)$ & $\hat{\alpha}$ & $0.0034(0.0010)$ & - \\
\hline & & & $\hat{\beta}$ & $0.0727(0.0062)$ & $0.0293(0.0025)$ \\
\hline & & & $\hat{\gamma}$ & $0.9755(0.0024)$ & $0.9670(0.0033)$ \\
\hline \multirow{3}{*}{8 hours } & $\hat{\rho}$ & $0.4267(0.0096)$ & $\hat{\alpha}$ & $0.0090(0.0029)$ & - \\
\hline & & & $\hat{\beta}$ & $0.0987(0.0122)$ & $0.0474(0.0056)$ \\
\hline & & & $\hat{\gamma}$ & $0.9609(0.0058)$ & $0.9376(0.0087)$ \\
\hline \multirow{3}{*}{12 hours } & $\hat{\rho}$ & $0.4358(0.0117)$ & $\hat{\alpha}$ & $0.0091(0.0037)$ & - \\
\hline & & & $\hat{\beta}$ & $0.1034(0.0161)$ & $0.0372(0.0064)$ \\
\hline & & & $\hat{\gamma}$ & $0.9597(0.0076)$ & $0.9541(0.0095)$ \\
\hline \multirow{3}{*}{1 day } & $\hat{\rho}$ & $0.4612(0.0159)$ & $\hat{\alpha}$ & $0.0105(0.0058)$ & - \\
\hline & & & $\hat{\beta}$ & $0.1031(0.0186)$ & $0.0497(0.0102)$ \\
\hline & & & $\hat{\gamma}$ & $0.9559(0.0097)$ & $0.9282(0.0178)$ \\
\hline
\end{tabular}

Notes: Parameter estimates and asymptotic standard errors for the three Gaussian copula models, non-dynamic, with Fisher transformation dynamic correlation and with Tse-Tsui dynamic correlation, fitted to the hourly up to daily returns on EUR/USD and JPY/USD rates. 
Table 11: Properties of the AR-BEKK model residuals

\begin{tabular}{lcccccccc}
\hline \multirow{2}{*}{$\begin{array}{l}\text { Time } \\
\text { horizon }\end{array}$} & \multicolumn{2}{c}{ EUR/USD } & & \multicolumn{2}{c}{ JPY/USD } & & \multicolumn{2}{c}{ A-D test } \\
\cline { 2 - 3 } 1 hour & $z_{t}$ & $z_{t}^{2}$ & & $z_{t}$ & $z_{t}^{2}$ & & EUR/USD & JPY/USD \\
\hline hours & 0.0592 & 0.0000 & & 0.0001 & 0.0000 & & 0.0000 & 0.0000 \\
4 hours & 0.1873 & 0.2105 & & 0.0733 & 0.7100 & & 0.0002 & 0.2289 \\
8 hours & 0.2752 & 0.0002 & & 0.7899 & 0.0107 & & 0.0106 & 0.1264 \\
12 hours & 0.2919 & 0.1796 & & 0.1956 & 0.4111 & & 0.0105 & 0.1695 \\
1 day & 0.7459 & 0.0939 & & 0.1069 & 0.7605 & & 0.0003 & 0.0000 \\
\hline
\end{tabular}

Notes: The first four left columns have the Ljung-Box autocorrelation tests pvalues up to the order twelve for the EUR/USD and JPY/USD residuals, $z_{t}$, and squared residuals, $z_{t}^{2}$, at the six time horizons. Columns five and six have the pvalues from the Anderson-Darling (A-D) goodness of fit test. The null hypothesis is that the residuals follow a Student- $t$ distribution. 
Table 12: Skewness, kurtosis, and correlation of the AR-BEKK model residuals

\begin{tabular}{lccccc}
\hline \multirow{2}{*}{ Time horizon } & \multicolumn{2}{c}{ EUR/USD } & \multicolumn{2}{c}{ JPY/USD } & Linear \\
& Skewness & Kurtosis & Skewness & Kurtosis & correlation \\
\hline 1 hour & -0.1218 & 6.897 & -0.1625 & 6.255 & 0.0142 \\
2 hours & -0.0248 & 4.702 & -0.1773 & 5.056 & 0.0210 \\
4 hours & -0.2767 & 5.892 & 0.0152 & 5.365 & -0.0165 \\
8 hours & -0.0794 & 2.066 & -0.2988 & 2.981 & -0.0174 \\
12 hours & -0.0272 & 1.576 & -0.3870 & 2.355 & -0.0084 \\
1 day & 0.0594 & 0.362 & -0.3462 & 1.776 & -0.0134 \\
\hline
\end{tabular}

Notes: Sample skewness and kurtosis of the EUR/USD and JPY/USD returns at the six time horizons. The right column displays the linear correlation between the EUR/USD and JPY/USD residuals. 
Table 13: Model evaluation on estimating correlation for different time horizons

\begin{tabular}{|c|c|c|c|}
\hline & $b_{0}$ & $b_{1}$ & $R^{2}$ \\
\hline \multicolumn{4}{|l|}{1 hour } \\
\hline Gauss Fisher & $-0.075(0.001)$ & $1.161(0.002)$ & 0.775 \\
\hline Gauss Tse-Tsui & $-0.015(0.001)$ & $1.256(0.003)$ & 0.780 \\
\hline$t$ Fisher & $-0.059(0.001)$ & $1.221(0.003)$ & 0.748 \\
\hline$t$ Tse-Tsui & $-0.081(0.001)$ & $1.177(0.003)$ & 0.737 \\
\hline BEKK & $-0.051(0.001)$ & $1.128(0.002)$ & 0.800 \\
\hline \multicolumn{4}{|l|}{2 hours } \\
\hline Gauss Fisher & $-0.090(0.001)$ & $1.193(0.003)$ & 0.817 \\
\hline Gauss Tse-Tsui & $-0.025(0.001)$ & $1.272(0.003)$ & 0.838 \\
\hline$t$ Fisher & $-0.074(0.001)$ & $1.158(0.003)$ & 0.814 \\
\hline$t$ Tse-Tsui & $-0.099(0.001)$ & $1.224(0.004)$ & 0.793 \\
\hline BEKK & $-0.041(0.001)$ & $1.106(0.003)$ & 0.814 \\
\hline \multicolumn{4}{|l|}{4 hours } \\
\hline Gauss Fisher & $-0.111(0.001)$ & $1.246(0.003)$ & 0.894 \\
\hline Gauss Tse-Tsui & $-0.033(0.001)$ & $1.290(0.004)$ & 0.883 \\
\hline$t$ Fisher & $-0.098(0.001)$ & $1.214(0.003)$ & 0.894 \\
\hline$t$ Tse-Tsui & $-0.141(0.002)$ & $1.318(0.004)$ & 0.870 \\
\hline BEKK & $-0.058(0.002)$ & $1.155(0.004)$ & 0.843 \\
\hline \multicolumn{4}{|l|}{8 hours } \\
\hline Gauss Fisher & $-0.140(0.002)$ & $1.326(0.006)$ & 0.886 \\
\hline Gauss Tse-Tsui & $-0.045(0.003)$ & $1.279(0.007)$ & 0.824 \\
\hline$t$ Fisher & $-0.126(0.002)$ & $1.283(0.005)$ & 0.885 \\
\hline$t$ Tse-Tsui & $-0.160(0.003)$ & $1.369(0.007)$ & 0.849 \\
\hline BEKK & $-0.065(0.003)$ & $1.131(0.007)$ & 0.804 \\
\hline \multicolumn{4}{|l|}{12 hours } \\
\hline Gauss Fisher & $-0.134(0.003)$ & $1.288(0.007)$ & 0.884 \\
\hline Gauss Tse-Tsui & $-0.070(0.003)$ & $1.347(0.008)$ & 0.856 \\
\hline$t$ Fisher & $-0.124(0.003)$ & $1.258(0.006)$ & 0.892 \\
\hline$t$ Tse-Tsui & $-0.185(0.004)$ & $1.396(0.008)$ & 0.865 \\
\hline BEKK & $-0.051(0.004)$ & $1.112(0.008)$ & 0.807 \\
\hline \multicolumn{4}{|l|}{ Daily } \\
\hline Gauss Fisher & $-0.136(0.006)$ & $1.315(0.012)$ & 0.848 \\
\hline Gauss Tse-Tsui & $-0.073(0.007)$ & $1.286(0.016)$ & 0.759 \\
\hline$t$ Fisher & $-0.164(0.006)$ & $1.341(0.011)$ & 0.865 \\
\hline$t$ Tse-Tsui & $-0.201(0.008)$ & $1.422(0.015)$ & 0.801 \\
\hline BEKK & $-0.021(0.008)$ & $1.053(0.016)$ & 0.668 \\
\hline
\end{tabular}

Notes: Quality assessment of the competing models using the regression of the transformed Kendall's correlation on the dynamic copula models correlation estimates. 
Table 14: Model evaluation on estimating daily correlation using data from different frequencies

\begin{tabular}{|c|c|c|c|c|c|c|}
\hline & \multicolumn{3}{|c|}{$\rho_{\tau, t}=b_{0}+b_{1} \rho_{\text {model }, h, t}+u_{t}$} & \multicolumn{3}{|c|}{$\rho_{\mathrm{RC}, t}=b_{0}+b_{1} \rho_{\text {model }, h, t}+u_{t}$} \\
\hline & $b_{0}$ & $b_{1}$ & $R^{2}$ & $b_{0}$ & $b_{1}$ & $R^{2}$ \\
\hline \multicolumn{7}{|l|}{1 hour } \\
\hline Gauss-Fisher & $0.061(0.008)$ & $1.006(0.017)$ & 0.632 & $-0.036(0.010)$ & $1.072(0.023)$ & 0.517 \\
\hline Gauss Tse-Tsui & $0.121(0.007)$ & $1.066(0.019)$ & 0.610 & $0.029(0.009)$ & $1.131(0.025)$ & 0.610 \\
\hline$t$ Fisher & $0.073(0.008)$ & $1.064(0.018)$ & 0.619 & $-0.023(0.010)$ & $1.131(0.025)$ & 0.503 \\
\hline$t$ Tse-Tsui & $0.038(0.008)$ & $1.062(0.017)$ & 0.654 & $-0.034(0.011)$ & $1.069(0.024)$ & 0.477 \\
\hline BEKK & $0.136(0.009)$ & $0.848(0.019)$ & 0.486 & $-0.047(0.009)$ & $1.124(0.019)$ & 0.612 \\
\hline \multicolumn{7}{|l|}{2 hours } \\
\hline Gauss Fisher & $0.067(0.007)$ & $0.969(0.016)$ & 0.640 & $-0.008(0.011)$ & $0.980(0.023)$ & 0.466 \\
\hline Gauss Tse-Tsui & $0.138(0.007)$ & $0.980(0.018)$ & 0.588 & $0.054(0.010)$ & $1.018(0.024)$ & 0.453 \\
\hline$t$ Fisher & $0.077(0.007)$ & $0.945(0.015)$ & 0.644 & $0.004(0.011)$ & $0.952(0.022)$ & 0.465 \\
\hline$t$ Tse-Tsui & $0.050(0.008)$ & $1.014(0.016)$ & 0.643 & $-0.010(0.011)$ & $0.993(0.024)$ & 0.442 \\
\hline BEKK & $0.149(0.008)$ & $0.800(0.017)$ & 0.501 & $0.009(0.009)$ & $0.962(0.020)$ & 0.516 \\
\hline \multicolumn{7}{|l|}{4 hours } \\
\hline Gauss Fisher & $0.099(0.008)$ & $0.872(0.016)$ & 0.587 & $0.026(0.011)$ & $0.878(0.022)$ & 0.425 \\
\hline Gauss Tse-Tsui & 0.167 (0.007) & $0.867(0.018)$ & 0.533 & $0.088(0.010)$ & $0.888(0.024)$ & 0.400 \\
\hline$t$ Fisher & $0.108(0.008)$ & $0.850(0.016)$ & 0.588 & $0.035(0.011)$ & $0.855(0.022)$ & 0.425 \\
\hline$t$ Tse-Tsui & $0.076(0.008)$ & $0.930(0.017)$ & 0.580 & $0.012(0.012)$ & $0.912(0.024)$ & 0.399 \\
\hline BEKK & $0.148(0.008)$ & $0.784(0.017)$ & 0.514 & $0.047(0.010)$ & $0.853(0.021)$ & 0.438 \\
\hline \multicolumn{7}{|l|}{8 hours } \\
\hline Gauss Fisher & $0.082(0.008)$ & $0.972(0.018)$ & 0.578 & $0.024(0.012)$ & $0.941(0.026)$ & 0.387 \\
\hline Gauss Tse-Tsui & $0.171(0.008)$ & $0.874(0.021)$ & 0.467 & $0.092(0.011)$ & $0.895(0.027)$ & 0.348 \\
\hline$t$ Fisher & $0.093(0.008)$ & $0.938(0.018)$ & 0.572 & $0.033(0.011)$ & $0.912(0.025)$ & 0.387 \\
\hline$t$ Tse-Tsui & $0.090(0.009)$ & $0.952(0.021)$ & 0.500 & $0.019(0.013)$ & $0.948(0.028)$ & 0.353 \\
\hline BEKK & $0.127(0.008)$ & $0.847(0.017)$ & 0.544 & $0.049(0.011)$ & $0.861(0.023)$ & 0.403 \\
\hline \multicolumn{7}{|l|}{12 hours } \\
\hline Gauss Fisher & $0.033(0.007)$ & $1.013(0.014)$ & 0.705 & $0.030(0.012)$ & $0.858(0.025)$ & 0.360 \\
\hline Gauss Tse-Tsui & $0.092(0.007)$ & $1.034(0.016)$ & 0.653 & $0.087(0.012)$ & $0.859(0.027)$ & 0.320 \\
\hline$t$ Fisher & $0.031(0.006)$ & $1.011(0.013)$ & 0.745 & $0.038(0.012)$ & $0.836(0.024)$ & 0.362 \\
\hline$t$ Tse-Tsui & $-0.014(0.007)$ & $1.113(0.015)$ & 0.712 & $0.012(0.014)$ & $0.896(0.028)$ & 0.327 \\
\hline BEKK & $0.098(0.007)$ & $0.872(0.014)$ & 0.635 & $0.066(0.011)$ & $0.785(0.022)$ & 0.370 \\
\hline \multicolumn{7}{|l|}{ Daily } \\
\hline Gauss Fisher & $-0.136(0.006)$ & $1.315(0.012)$ & 0.848 & $-0.047(0.015)$ & $0.975(0.030)$ & 0.330 \\
\hline Gauss Tse-Tsui & $-0.073(0.007)$ & $1.286(0.016)$ & 0.759 & $-0.005(0.015)$ & $0.966(0.032)$ & 0.304 \\
\hline$t$ Fisher & $-0.164(0.006)$ & $1.341(0.011)$ & 0.865 & $-0.065(0.016)$ & $0.989(0.031)$ & 0.333 \\
\hline$t$ Tse-Tsui & $-0.201(0.008)$ & $1.422(0.015)$ & 0.801 & $-0.095(0.017)$ & $1.055(0.034)$ & 0.312 \\
\hline BEKK & $-0.021(0.008)$ & $1.053(0.016)$ & 0.668 & $-0.020(0.014)$ & $0.902(0.027)$ & 0.348 \\
\hline
\end{tabular}

Notes: Quality assessment of the competing models using the daily transformed Kendall's correlation and realized correlation. The daily correlation estimates are obtained from the copula modeling at different time horizons. The non-parametric estimates are then projected on the parametric estimates. 


\section{Figure captions:}

Figure 1: Time-varying cross-correlations estimated by Kendall's tau and by the timevarying copula-based models Student- $t$ and Gaussian both with Fisher transform for the daily returns on the EUR/USD and JPY/USD spot rates.

Figure 2: Regression of the estimates of Kendall's tau for the filtered returns, computed according to (11), on the dynamic copula-GARCH and BEKK correlation estimates. 
Figure 1:

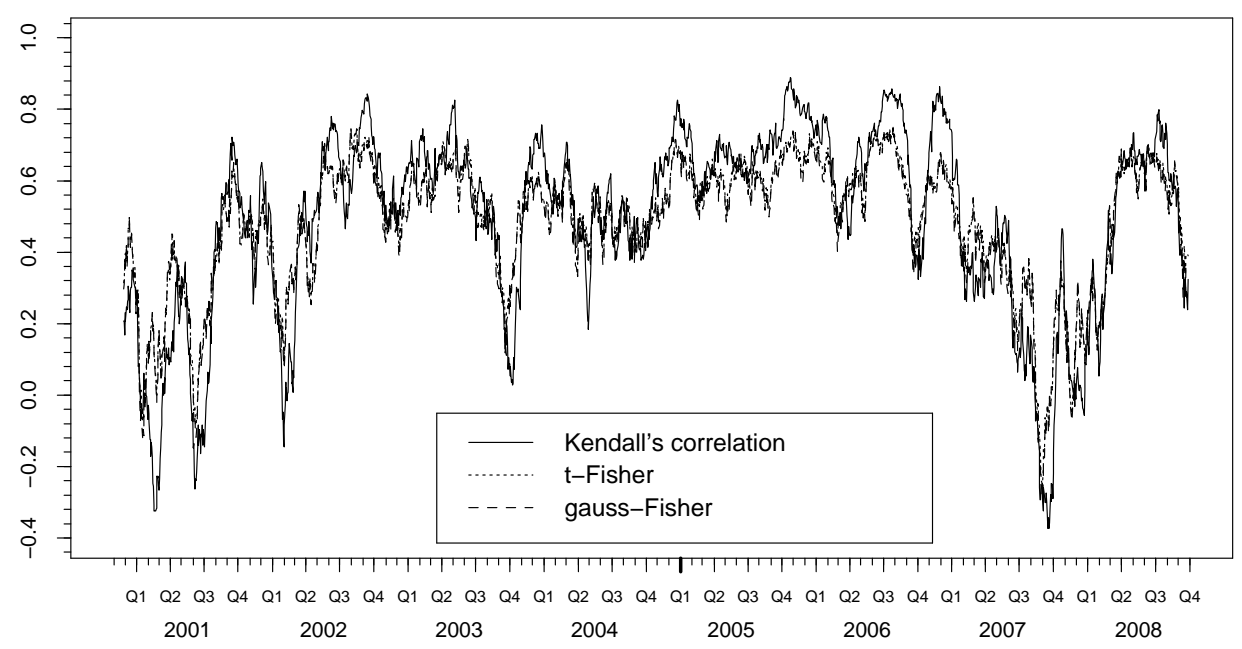


Figure 2:
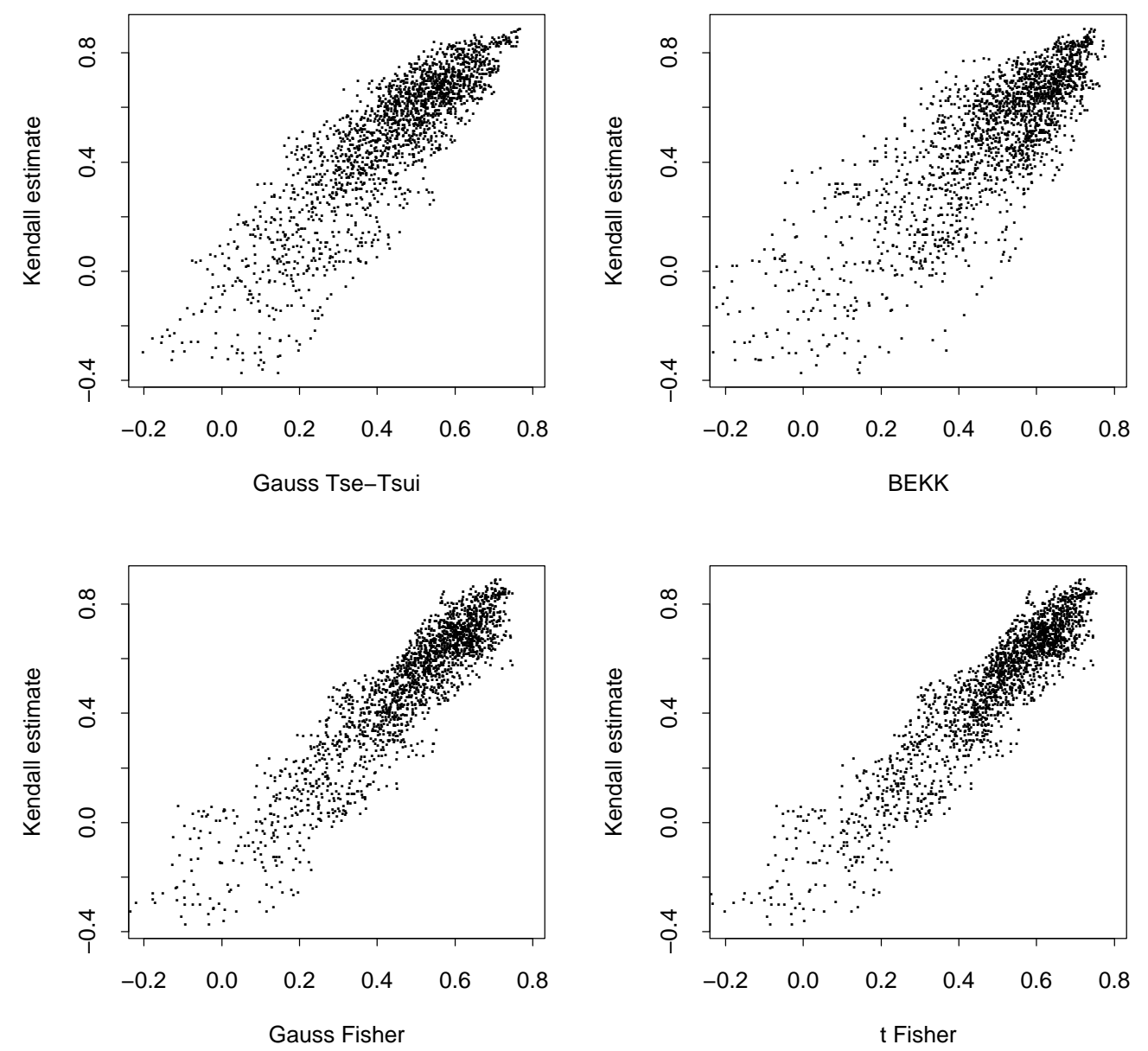